\title{
Reconstruction of the large multi-aperture beam via IR calorimetry technique and beam emission spectroscopy at the ELISE test facility
}

\author{
Isabella Mario, Federica Bonomo, Dirk Wünderlich, Ursel \\ Fantz, Riccardo Nocentini \\ Max Planck Institute for Plasma Physics, Boltzmannstr. 2, 85748 Garching (DE) \\ E-mail: isabella.mario@ipp.mpg.de
}

\begin{abstract}
The ELISE test facility with its half ITER size ion source is an important intermediate step in the European development roadmap toward the ITER neutral beam injection system. Of particular interest is the beam characterization at ELISE in terms of homogeneity and divergence. The two main beam diagnostic systems are beam emission spectroscopy (BES), providing beam intensity and divergence and infrared (IR) calorimetry applied to the surface of a diagnostic calorimeter, which provides a spatially resolved 2D map of the beam power density. Beam parameters such as width, intensity, position and accelerated current are retrieved by a fitting routine which is validated using synthetic beam profiles, thus demonstrating that the beam parameters are correctly reproduced. By comparing results of BES and IR calorimetry for several thousand pulses, it is demonstrated that the beam width is, to a large extent, determined by the beam divergence. BES and IR calorimetry are then combined to reconstruct the beam profile and this is the basis to investigate procedures for improving the beam quality in terms of power density homogeneity.
\end{abstract}

\section{Introduction}

The future international fusion experiment ITER ("The Way" in Latin) will be equipped with two $1 \mathrm{MeV} \mathrm{D}_{0}$ or $870 \mathrm{keV} \mathrm{H}_{0}$ heating neutral beam injectors (HNBI) for a total power of $33.3 \mathrm{MW}$ and with one $100 \mathrm{keV}$ diagnostic neutral beam (DNB) [1]. In these systems, negative hydrogen or deuterium ions are extracted from a large source $(2 \mathrm{~m} \times 1 \mathrm{~m})$ and accelerated in a multi-grid, multi-aperture electrostatic accelerator. The large (about $1.6 \mathrm{~m}$ height and $0.8 \mathrm{~m}$ width) negative ion beam is then neutralized in a gas neutralizer and the residual charged particles are electrostatically removed from the beamline. To ensure transmission through a $1.08 \mathrm{~m}$ high, $0.55 \mathrm{~m}$ wide port placed at about $26 \mathrm{~m}$ distance from the ion source, the grids are tilted, thus focusing the beam [1][2]. The ITER requirements for the NBI system in terms of extracted current density are $286 \mathrm{~A} / \mathrm{m}^{2}$ for $\mathrm{D}^{-}\left(329 \mathrm{~A} / \mathrm{m}^{2}\right.$ for $\left.\mathrm{H}^{-}\right)$, with an electron-to-ion ratio 
lower than one, to minimize the power load deposition on beamline components. Source pressure is restricted to $0.3 \mathrm{~Pa}$ to minimize the stripping losses, namely loss of negative ions neutralized before being fully accelerated, to less than $30 \%$. The pulse length is up to one hour in deuterium (1000 s in hydrogen) [1][3]. The source is foreseen to deliver a total accelerated current density of $200 \mathrm{~A} / \mathrm{m}^{2}$ for $\mathrm{D}^{-}\left(230 \mathrm{~A} / \mathrm{m}^{2}\right.$ for $\left.\mathrm{H}^{-}\right)$before the neutralization stage. Strict requirements on the beam properties have to be fulfilled to maximize the beam transmission through the beamline and to minimize the power loads on the beamline components: the core beamlet divergence, i.e. the width of the angular velocity distribution of the negative ions, must be less than $7 \mathrm{mrad}$ with a power homogeneity among beamlets better than $90 \%$ [1][4].

In the framework of the European roadmap toward the ITER neutral beam injection system [5], the ELISE (Extraction from a Large Ion source Experiment) test facility $[6][7]$ is a fundamental step towards the full size NBI systems. The ELISE ion source has half of the height and the same width of the ITER NBI source. The testbed aims to demonstrate the simultaneous achievement of the ITER requirements for the ion source [3]. The ELISE extraction and acceleration system is made of three grids: the plasma grid (PG) which directly faces the plasma, the extraction grid (EG), and the grounded grid (GG). The grids are flat and the apertures are aligned, thus no beam focusing or beamlet steering is applied. A horizontal magnetic field, called filter field, is created by a current $\mathrm{I}_{\mathrm{PG}}$ flowing vertically through the $\mathrm{PG}$. For $\mathrm{I}_{\mathrm{PG}}=1 \mathrm{kA}$, the filter field strength is $0.95 \mathrm{mT}$ at $2 \mathrm{~cm}$ axial distance from the PG. The filter field is employed to reduce the electron temperature and density and, combined with pressure gradients and electric fields, it results in vertical plasma drifts which affect the vertical profile of the plasma density and of the plasma potential [8]. The filter field is present also within and downstream of the grid system, thus affecting the beam particle trajectories. Permanent magnets, called deflection magnets, are embedded in the EG to dump the co-extracted electrons on the grid itself. The trajectories of the negative ions are also affected by the deflection magnets. Beamlet compensation, as foreseen for the ITER grid system [1], is not applied at ELISE. The extraction system is based on the SINGAP design [9], with a modified design for the grids to maximize the extracted negative ion current density for an extraction voltage of about $10 \mathrm{kV}[10]$ and to adapt the gap between EG and GG to the total high voltage available, i.e. up to $60 \mathrm{kV}$.

For a $\mathrm{D}^{-}$extracted current density of $286 \mathrm{~A} / \mathrm{m}^{2}$ (ITER target), the beamlet divergence, defined as half width at one e-folding of the angular particle distribution, predicted at ELISE by the IBSIMU code [11][12] is $14.4 \mathrm{mrad}$ (19.5 mrad for an $\mathrm{H}^{-}$extracted current density of $\left.329 \mathrm{~A} / \mathrm{m}^{2}\right)$. The simulations are performed with a perpendicular particle velocity of $1 \mathrm{eV}$ [13], $10 \mathrm{kV}$ of extraction voltage, and $50 \mathrm{kV}$ of acceleration voltage. The arrangement of the 640 circular apertures (aperture diameter of $14 \mathrm{~mm}$, total extraction area of $985 \mathrm{~cm}^{2}$ ) on the PG is organized in 8 rectangular beamlet groups of $16 \times 5$ apertures each [6]. The beamlet group structure is similar to that of the ITER extraction system. The grids are vertically divided into two segments, each one hosting four beamlet groups (see figure 1 or 6(a)). The beam portions created by these grid 
segments are called top and bottom beam segments, respectively. The beam is stopped by a diagnostic calorimeter placed at $3.5 \mathrm{~m}$ distance from the GG [14]. The two main beam diagnostics used to study the beam divergence and power density profile are beam emission spectroscopy (BES) and infra-red (IR) calorimetry applied to the diagnostic calorimeter.

The characterization of the beam is of high relevance to determine and study the homogeneity of the beam power density and this will serve as basis for developing procedures and recipes to improve the beam homogeneity. In this work BES and IR calorimetry are described and the data analysis is discussed. For IR calorimetry the calibration procedure, which allows the retireval of the absolute beam power deposited, is introduced and described for the first time. BES and IR calorimetry are crosschecked and then combined to reconstruct the beam properties.

\section{Overview of the beam diagnostics}

In ELISE, the total extracted negative ion current $I_{\text {ex }}$ is measured electrically and the total accelerated current deposited on the diagnostic calorimeter is measured by IR calorimetry and water calorimetry. The following beam diagnostics are installed downstream of the grid system, as shown in figure 1: the tungsten wire (W-wires) calorimeter, the BES diagnostics and the diagnostic calorimeter [15]. The tungsten wire calorimeter consists of a frame of tungsten wires drawn vertically and horizontally in a plane perpendicular to the beam direction at $1.8 \mathrm{~m}$ axial distance from the GG [15]. This diagnostic is not absolutely calibrated, thus providing only qualitative monitoring images of the beam power deposited onto the wires. At about $2.7 \mathrm{~m}$ downstream of the GG, 20 BES lines of sight (LOS) [15] measure the beam, providing local but line-integrated measurements of beam divergence, intensity and stripping losses. The diagnostic is based on Doppler-shifted $H_{\alpha}$ emission originating from collisions between beam particles and background gas [8]. The beam is stopped at $3.5 \mathrm{~m}$ from the grids by a diagnostic calorimeter [15] designed to perform calorimetric and thermometric measurements of the deposited beam power.

Since the beam is measured by BES and by the diagnostic calorimeter at large distances from the GG, single beamlet measurements are not possible due to the large beamlet overlap occurring at such axial distances. The beam divergence can be considered a fixed value once space charge compensation [16] occurs, thus preventing the sudden increase of beamlet divergence due to electrostatic repulsion of the beam particles. Space charge compensation depends on the background gas density (about 0.01-0.05 Pa at ELISE) and on the beamlet current density. At ELISE, in case of good beam optics, the beam divergence measured by BES diagnostics is around 1.5 to 2 degrees (26 to $35 \mathrm{mrad}$ ). The measured value is higher than the predicted one, i.e. between 14.4 and $19.5 \mathrm{mrad}$, because the overlap of multiple rows of beamlets, each one horizontally deflected alternately left or right by the deflection magnets, causes an artificial broadening of the line-integrated Doppler peak seen by the horizontal LOS. The effect of the beamlet overlapping on 


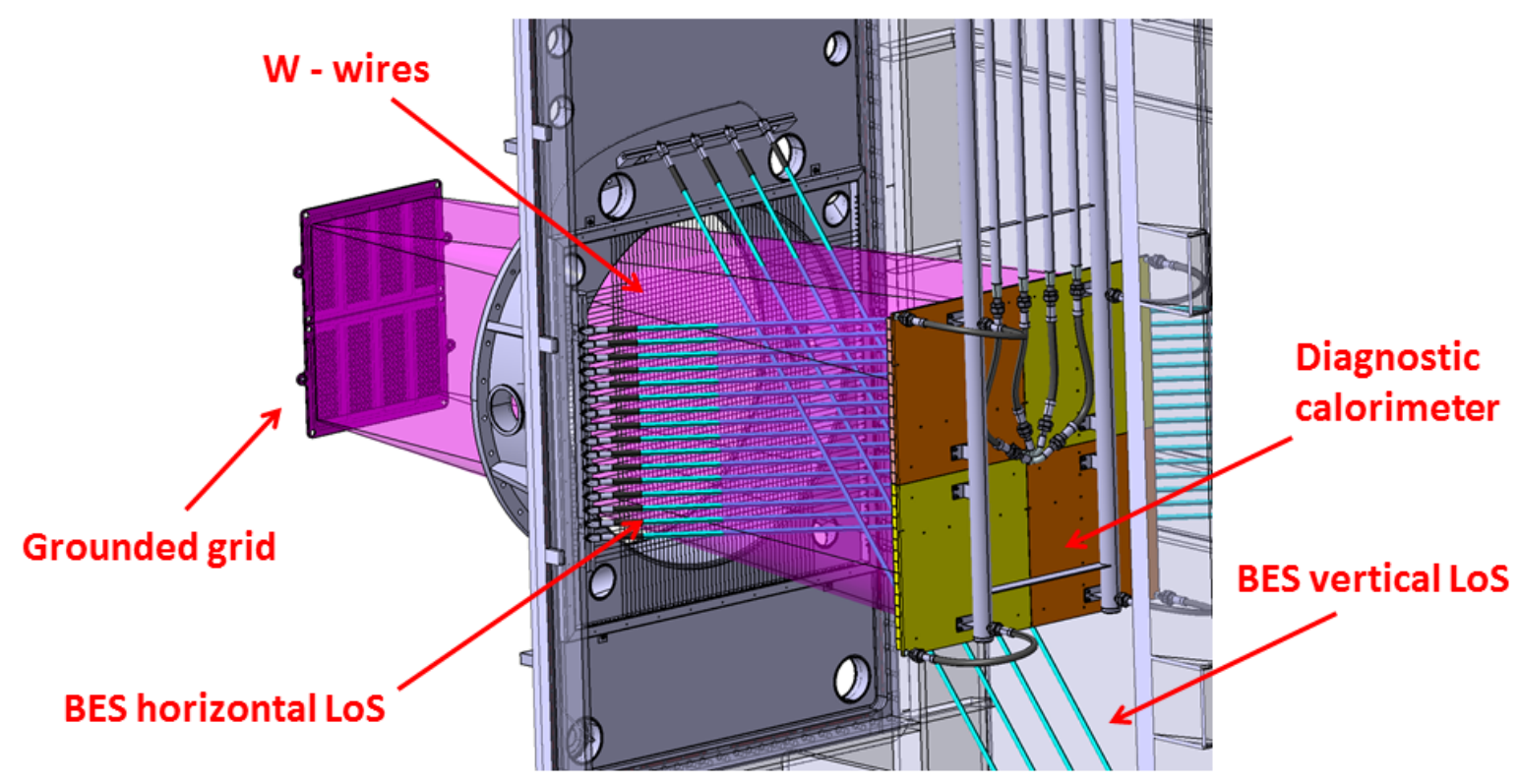

Figure 1: Overview of the beam diagnostic systems at ELISE [15]: the W-wire calorimeter, the two arrays of lines of sight (LOS) of the BES diagnostic and the diagnostic calorimeter on the right. On the leftmost side of the figure, the downstream side of the grounded grid is shown.

the broadening of Doppler peak is shown in reference [17] calculated for the Batman Upgrade test facility [18][19]. The beam position is influenced by the filter field which is still considerably intense downstream of the GG, e.g. for $I_{\mathrm{PG}}=1 \mathrm{kA}$ at $0.5 \mathrm{~m}$ downstream of the $\mathrm{PG}$ the $\mathrm{FF}$ is $0.15 \mathrm{mT}$, thus resulting in a net Lorentz force displacing the beam downwards in the present configuration. The displacement, being usually between $2 \mathrm{~cm}$ and $10 \mathrm{~cm}$ measured at the calorimeter, depends on the strength of the filter field, on the total high voltage and on the used hydrogen isotope. The beam power profile mimic the arrangement of the beamlet groups on the GG: the beam is vertically divided in two segments which usually differ in intensity and width. For each beam segment, the four beamlet groups can be distinguished or not according to the beam divergence. The two central beamlet groups can be more intense due to the overlap of the neighboring groups.

\section{Beam emission spectroscopy}

The BES diagnostic is based on the Doppler shift effect of the $\mathrm{H}_{\alpha}$ or $\mathrm{D}_{\alpha}$ Balmer line produced by impact of the accelerated beam particles with background particles. The most relevant reactions for the production of $\mathrm{H}_{\alpha}$ are [8],

$$
\begin{gathered}
\underline{H^{-}}+H_{2} \rightarrow \underline{H^{0}(n=3)}+H_{2}+e \\
\underline{H^{0}}+H_{2} \rightarrow \underline{H^{0}(n=3)}+H_{2} \\
\underline{H^{-}}+H_{2} \rightarrow \underline{H^{0}+2 H^{0}(n=3)}
\end{gathered}
$$


where the fast particles are underlined. The processes are the same in case of deuterium. The light is collected by 20 optic heads placed inside the vacuum chamber as shown in figure 1: the light collected by each of them is conveyed through an optical fiber to a high-resolution Acton spectrometer equipped with a CCD camera (the dispersion is 7 $\mathrm{pm} /$ pixel for a spectral interval of $8 \mathrm{~nm}$ ). The optical head axis has an angle $\zeta=50^{\circ}$ with respect to the beam direction and the radiation emitted is Doppler shifted of the quantity,

$$
\lambda_{\text {Doppler }}=\frac{(1+\beta \cos \zeta)}{\sqrt{1-\beta^{2}}} \lambda_{0},
$$

where $\beta=v / c$ represents the particle velocity and $\lambda_{0}$ is the wavelength for the unshifted $\mathrm{H}_{\alpha}$ or $\mathrm{D}_{\alpha}$ line. The opening angle of the observation cone for the optical head has been measured to be $0.38^{\circ}$, which is in agreement with the value calculated by taking into account the geometry of the optical system, the fiber and the lens properties. The optic heads are positioned at about one meter distance from the geometrical beam center and, by assuming a beam width of $1 \mathrm{~m}$, the viewing cone has a diameter of about $20 \mathrm{~mm}$ at the entrance and about $32 \mathrm{~mm}$ at the exit of the beam region. Therefore, the signal collected has to be assigned to several rows of beamlets, every row alternately deflected left or right. For every beamlet, the signal is collected at different distances from the GG depending on the position of the beamlet along the LOS. These effects together increase the broadening of the Doppler peak, resulting in an increased value of measured divergence, which is not representative of the beamlet divergence but rather of an averaged value over the beam width. The beam divergence is sensitive to changes of the beam optics.

A typical BES spectrum, acquired with an exposure time of $1.5 \mathrm{~s}$, is shown in figure 2(a), where three main features are distinguishable: the $H_{\alpha}$ unshifted peak on the left, the stripping peak caused by negative ions neutralized by collisions with the background gas before being fully accelerated, and the Doppler shifted $H_{\alpha}$ peak at higher wavelength. The Doppler peak integral is proportional to the averaged beam current density and to the background gas density.

The Doppler peak half width at one e-folding $\Delta \lambda$ is determined through a Gaussian fit applied to the Doppler peak (fit applied to the signal above $30 \%$ of the peak) and the beam divergence $\vartheta$ is calculated from $\Delta \lambda$, also taking into account the apparatus profile of the spectrometer and the opening angle of the lens head. The line broadening of the Doppler peak $\Delta \lambda$ is determined by the spectrometer instrumental function $\Delta \lambda_{N}$, by the opening angle of the observation cone for the optical head $\omega$ and by the divergence $\vartheta$ (see equation $2[21]$ ),

$$
\Delta \lambda^{2}=\Delta \lambda_{N}^{2}+\left(\frac{\lambda_{0}}{\sqrt{1-\beta^{2}}} \beta \sin \zeta\right)^{2}\left(\omega^{2}+\vartheta^{2}\right) .
$$

In ELISE and for the used spectrometer $\Delta \lambda_{N}=0.02522 \mathrm{~nm}, \omega=0.38^{\circ}$ [20]. The apparatus profile of the spectrometer is an order of magnitude lower than the Doppler 

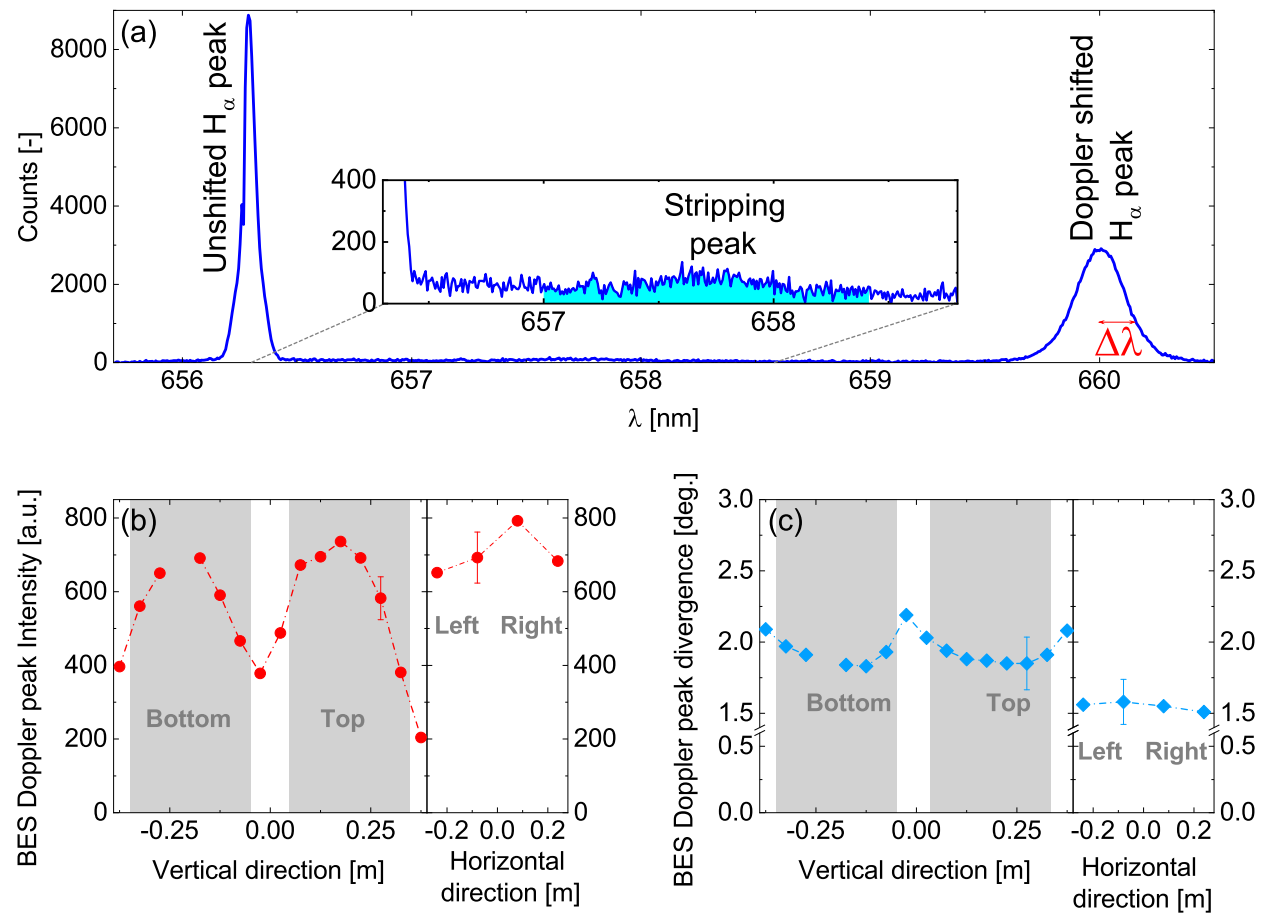

Figure 2: (a) Example spectrum from one LOS (LOS 10 positioned at $+7.5 \mathrm{~cm}$ from the geometrical center of the beam) of the BES diagnostic: at $656.28 \mathrm{~nm}$ the $H_{\alpha}$ unshifted peak is visible while the Doppler shifted peak is placed at a wavelength shift proportional to the total HV applied. Between these two peaks, the stripping peak is visible. Vertical and horizontal profiles of the Doppler peak integral are shown in (b). Vertical and horizontal profiles of the divergence retrieved from the Doppler peak width are shown in (c). In (b) and (c), shaded areas correspond to the vertical projection of the beamlet groups. The LOS positioned at $-0.225 \mathrm{~m}$ is not available. Shot 27115 performed in hydrogen at $0.3 \mathrm{~Pa}$ filling pressure, $180 \mathrm{~kW}$ total $\mathrm{RF}$ power for an extracted current of $\mathrm{I}_{\mathrm{ex}}=18.7 \mathrm{~A}$ with $6 \mathrm{kV}$ extraction and $30 \mathrm{kV}$ acceleration voltage; $\mathrm{I}_{\mathrm{PG}}=1.6 \mathrm{kA}$.

peak width typically measured in the experiment. Furthermore, the natural line broadening is negligible compared to the Doppler broadening. Therefore, the main contribution to the line is the broadening due to the particle angular distribution. For the estimation of the errors, the different sources of error as listed in ref. [22] contribute to an error of about $10 \%$ on the LOS-integrated beam divergence value and the main contribution to the error is the statistical error on the raw signal. The HV ripple, which can cause variations of the extracted current density and, consequently of the divergence, is small, i.e. about $100 \mathrm{~V}$ over an extraction voltage of $4 \mathrm{kV}$ or more. The contribution to the Doppler peak broadening of the HV ripple, over which the BES spectrum is integrated, is negligible.

16 LOS are looking along the horizontal direction, thus giving a vertical profile while four LOS are looking vertically providing a horizontal profile. The horizontal LOS positioned at $-22.5 \mathrm{~cm}$ is not available because the fiber coupling to the spectrometer is damaged. The spacing is $5 \mathrm{~cm}$ between the horizontal lens heads and $16 \mathrm{~cm}$ between the vertical ones: a higher resolution is desirable along the vertical direction to identify the effect 
of the plasma drift $[8]$ on the beam profile. The LOS are relatively calibrated, so it is possible to compare the absolute intensity given by each LOS. In figure 2(b) and 2(c) the vertical and horizontal beam intensity and profile are shown, respectively. The vertical profile of the Doppler peak integral shown in figure 2(b) highlights the presence of two areas with high intensity: these are the vertical projection of the top and bottom beam segments. For LOS measuring an higher current density, a lower divergence is observed as shown in figure 2(c). The LOS between the top and the bottom beam segments, in a portion of volume not directly illuminated by apertures, measure a low intensity with high beam divergence, indicating that the beam in this region is weak and formed by highly divergent particles originating from beamlets located on the beam segments. A better understanding of the origin of the signal measured by such LOS is possible only with codes such as BBCNI [17]. The beam is not centered with respect to the zero of the scale but slightly displaced downwards due to the vertical deflection caused by the filter field. The horizontal divergence (measured by the 16 horizontal LOS) is typically higher than the vertical one (measured by the four vertical LOS) due to broadening of the Doppler peak caused by the overlap of rows of beamlets which are left and right deflected.

\section{Infra-red calorimetry}

The calorimeter is made of four inertially cooled copper plates of dimension $60 \mathrm{~cm} \times 60 \mathrm{~cm} \times 2.5 \mathrm{~cm}$. A detailed description is done in reference [15]. The beam power deposited into each plate is measured separately by water calorimetry. 900 copper blocks, each of $3.8 \mathrm{~cm} \times 3.8 \mathrm{~cm}$ surface area, are brazed onto the water-cooled copper plates. The schematic of the $30 \times 30$ blocks is displayed in figure $3(\mathrm{a})$. A $2 \mathrm{~mm}$ gap separates the blocks such that each block can be considered as a single independent heat load measuring element. The power deposited on the blocks passes through an intermediate thermal resistance to the actively cooled plate: in this way the blocks can be considered as inertial calorimeters during the beam-on time. The heat loss due to radiation is negligible at the typical temperature reached by the blocks, and at the maximum temperature that the calorimeter can withstand without damage (about $450^{\circ} \mathrm{C}$ [15]) radiation losses are in the order of a few percent. 48 thermocouples, used to measure the absolute block temperature, are embedded into the same number of blocks as shown with blue squares in figure 3(a).

A high resolution $(640 \times 480$ pixels $)$ FLIR A655sc IR micro-bolometer camera placed outside the vacuum chamber looks at the diagnostic calorimeter through a $\mathrm{ZnSe}$ window with anti-reflective coating. To decrease the reflectivity of the beam-facing side of the diagnostic calorimeter, the surface has been covered with a $\mathrm{MoS}_{2}$ coating [14]. 

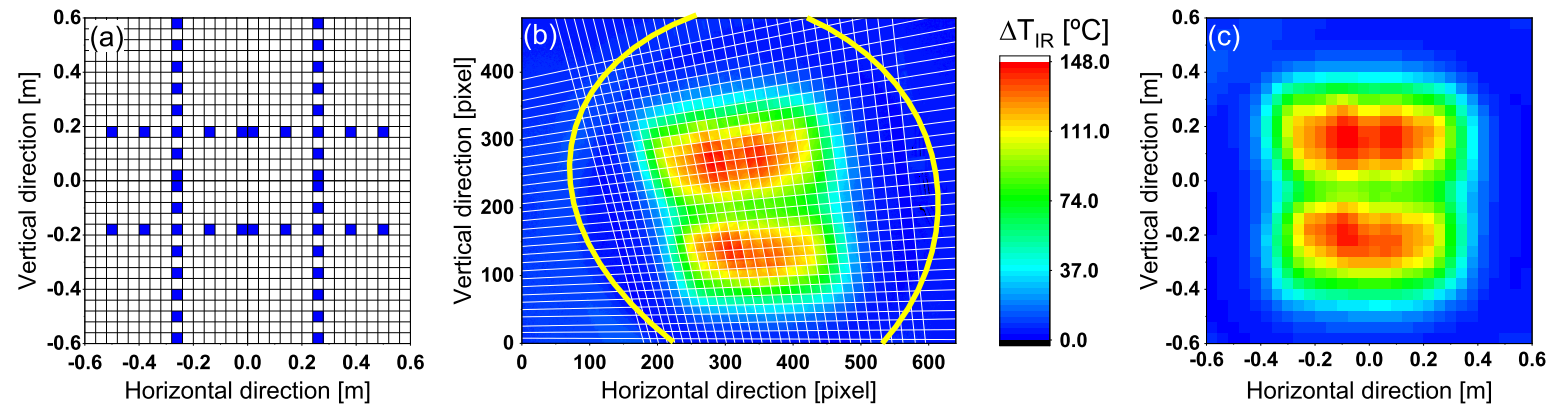

Figure 3: (a) Schematic of the 900 block composing the calorimeter surface. The blocks is which 48 thermocouples are embedded are indicated in blue. (b) $\Delta \mathrm{T}$ image as retrieved from the IR camera. The blocks are defined by the white lines while the yellow thicker lines indicate the limited field of view due to geometrical vignetting. The average temperature values inside each block are shown in (c): a $2 \mathrm{D}$ beam profile (resolution: $30 \times 30$ ): the spatial resolution is given by the block dimension of $3.8 \mathrm{~cm} \times 3.8 \mathrm{~cm}$. Data of shot 27115 performed in hydrogen at $0.3 \mathrm{~Pa}$ filling pressure, $180 \mathrm{~kW}$ total power for an extracted current of $\mathrm{I}_{\mathrm{ex}}=18.7 \mathrm{~A}$ with $6 \mathrm{kV}$ extraction and $30 \mathrm{kV}$ acceleration potential; $\mathrm{I}_{\mathrm{PG}}=1.6 \mathrm{kA}$.

\subsection{Camera calibration and temperature evaluation}

The total radiation seen by the IR camera detector is a function of the power emitted by the calorimeter surface $W_{\text {cal }}$, of the distance between emitting surface and camera, of the emissivity $\varepsilon$ of the calorimeter surface and of the transmissivity $\tau_{\text {opt }}$ of the ZnSe window placed between the IR camera and the calorimeter. The internal calibration coefficient of the micro-bolometer, $\varepsilon, \tau_{\text {opt }}$ and the wavelength response of the chip are used by the processing software to obtain the calorimeter surface temperature $T_{\text {cal }}$ from $W_{\text {cal }}$. The problem can be simplified by considering that only the temperature increase $\Delta T$ during the beam-on phase is required to evaluate the power density deposited. In figure 3(b) the temperature increase seen by the IR camera is shown: the straight thin lines show the blocks while the thick yellow lines indicate the field of view limited due to vignetting by the pipe. In figure $3(\mathrm{c})$ the temperature increase for each block is shown: the temperature values are retrieved by averaging over the pixels inside each block. Both the emissivity $\varepsilon$ and the transmissivity $\tau_{\text {opt }}$ can change in time: the emissivity $\varepsilon$ is mainly related to the status of the blackening layer, i.e. $\varepsilon$ can be reduced locally due to removal of coating by sputtering; the transmissivity $\tau_{\text {opt }}$ can change due to coating of the ZnSe window by the sputtered material from the calorimeter. It is not possible to monitor these two quantities continuously and without breaking the vacuum of the tank. Therefore, for simplicity, the transmissivity $\tau_{\text {opt }}$ and the emissivity $\varepsilon$ are combined into an effective emissivity $\varepsilon_{\text {eff }}=\varepsilon \tau_{\text {opt }}$, whose value is estimated experimentally by comparing the $\Delta T$ measured by the IR camera $\left(\Delta T_{\mathrm{IR}}\right)$ with the value obtained from the thermocouples $\left(\Delta T_{\mathrm{TC}}\right)$. For one block hosting a thermocouple, $\varepsilon_{\text {eff }}$ is varied within a range of values and $\Delta T_{I R}$ is calculated for each case: the value of $\varepsilon_{\text {eff }}$ which minimizes the quantity $\left|\Delta T_{\mathrm{IR}}-\Delta T_{\mathrm{TC}}\right|$ is then the best effective emissivity for the selected block. The evolution of $\left|\Delta T_{\mathrm{IR}}-\Delta T_{\mathrm{TC}}\right|$ as a function of $\varepsilon_{\text {eff }}$ is shown in figure $4(\mathrm{a})$. This procedure 
is repeated for the 4 blocks in the center of each calorimeter plate (as displayed in the schematic on the top right in figure 4(a)). The effective emissivity value for the whole surface of the calorimeter is an average of these four values. The error on the emissivity is determined by statistical error propagation taking into account the error of $\Delta T_{\mathrm{TC}}\left(2.2^{\circ}\right.$ $\mathrm{C}$ for the single thermocouple temperature value, $3.1^{\circ} \mathrm{C}$ for $\Delta T_{\mathrm{TC}}$ ) and the maximum error of $\Delta T_{\mathrm{IR}}$ for a fixed $\varepsilon$ (footnote $\ddagger, 2^{\circ} \mathrm{C}$ for the single absolute temperature, $2.8^{\circ} \mathrm{C}$ for $\left.\Delta T_{\mathrm{IR}}\right)$. The final error on the quantity $\left|\Delta T_{\mathrm{IR}}-\Delta T_{\mathrm{TC}}\right|$ is $4.2^{\circ} \mathrm{C}$. The error on $\varepsilon_{\mathrm{eff}}$ is \pm 0.05 and of \pm 0.03 on the averaged emissivity value. For the case shown in figure $4(\mathrm{a})$, the averaged value for the effective emissivity is $0.88 \pm 0.03$. The emissivity estimation is performed for each beam pulse. The temporal evolution of the effective emissivity can be monitored in time for every beam pulse to check possible anomalies in the calorimeter or in the IR evaluation. The temperature increase $\Delta T_{\mathrm{TC}}$ is calculated with respect to the

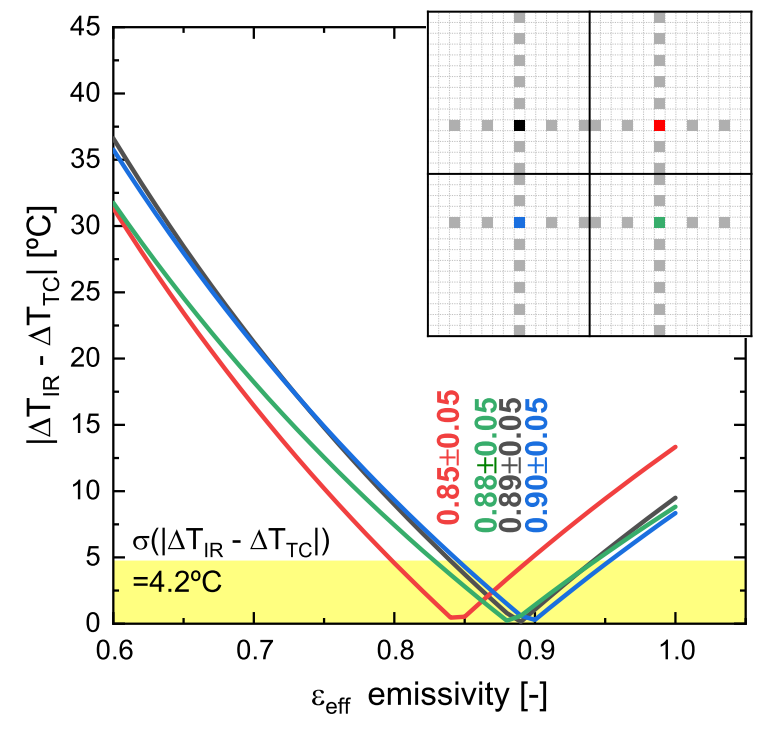

(a)

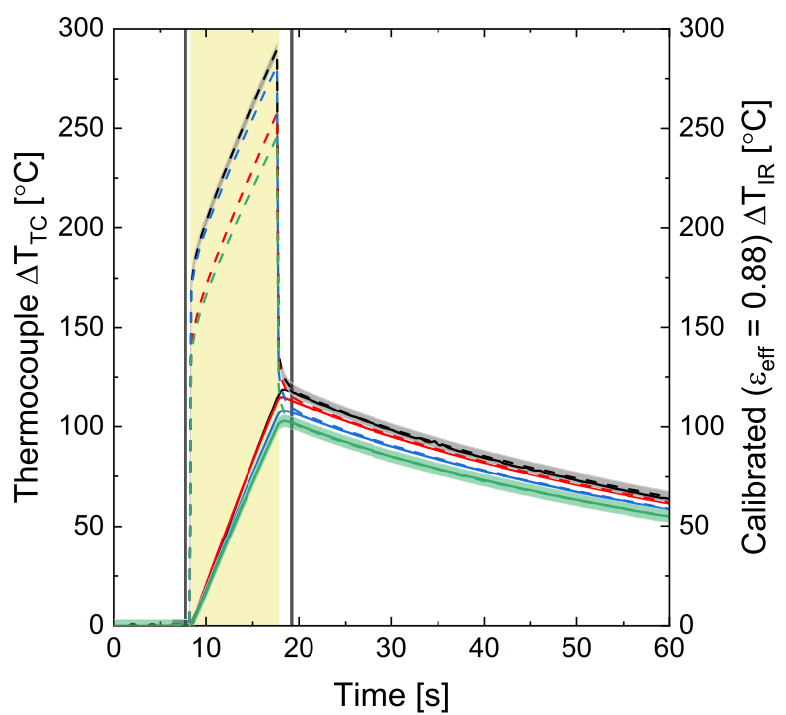

(b)

Figure 4: (a) Difference $\left|\Delta \mathrm{T}_{\mathrm{IR}}-\Delta \mathrm{T}_{\mathrm{TC}}\right|$ as a function of the effective emissivity for four thermocouples. Colors refer to the blocks as shown in the small schematic. The best effective emissivity value for each thermocouple is reported in figure. (b) Temperature difference $\Delta \mathrm{T}$ evolution in time; measurement from the thermocouples (full line) and from the calibrated IR camera (dashed line) measuring in the same blocks housing the thermocouples. The shaded area highlights the beam-on phase of $9.5 \mathrm{~s}$. The vertical black lines show the two times used for determining $\Delta \mathrm{T}_{\mathrm{IR}}$ before and after the extraction phase. The colors refer to the blocks as shown in the small schematic in (a). For two cases the error bar is contouring the data. Shot 27115 , the effective emissivity is calculated to be $\varepsilon_{\text {eff }}=0.88$. $\ddagger$ Infrared Camera Accuracy and Uncertainty in Plain Language, https://www.flir.com/discover/rdscience/infrared-camera-accuracy-and-uncertainty-in-plain-language/ 
corresponding $\Delta T_{\mathrm{IR}}$ by IR calorimetry (dashed lines) is shown. The beam-on phase is shaded while the two points in time for the determination of $\Delta T_{\mathrm{IR}}$ are shown by vertical black lines: at these times, once the $\varepsilon_{\text {eff }}$ calibration is correctly done, IR calorimetry and thermocouple temperature values superimpose. A significant discrepancy between IR measurements and thermocouples is observed during the beam-on phase (shown in figure 4). The most likely explanation is that the thin blackening layer heats up considerably more than the copper substrate due to bad thermal conductivity between the two [14]. This temporary additional increase of the temperature during the beam-on time phase has no impact on the evaluation of the total temperature increase $\Delta \mathrm{T}$ and, consequently, on the beam power estimation because the temperature is measured before the beam and immediately after this phenomenon vanishes. Although the camera has a high resolution, the actual experimental spatial resolution for a 2D map of the beam power deposited onto the calorimeter is given by the $30 \times 30$ calorimeter blocks as shown in $3(\mathrm{c})$. The temperature increase $\Delta T$ is proportional to the deposited beam power $P$,

$$
P=\frac{c_{\mathrm{Cu}} m \Delta T}{t_{B}},
$$

where $m$ is the mass of the single copper blocks, $c_{\mathrm{Cu}}$ is the heat capacity of copper and $t_{\mathrm{B}}$ is the beam-on time. For the case shown in figure 3(c), the power deposited in $9.5 \mathrm{~s}$ extraction phase for the block with the highest temperature increase is $(1.96 \pm 0.06) \mathrm{kW}$, the total power deposited onto the calorimeter is $586 \mathrm{~kW}$. From the total HV $(36.2 \mathrm{kV})$ and the total extraction area $\left(985 \mathrm{~cm}^{2}\right)$, an accelerated averaged current density of $164 \mathrm{~A} / \mathrm{m}^{2}$ is calculated.

A validation of the total power averaged over the calorimeter surface measured by IR analysis is done through a comparison with the water calorimetry measurement also averaged over the diagnostic calorimeter. The comparison of the total accelerated current density (obtained dividing the averaged power density by the applied HV) deposited onto the diagnostic calorimeter measured by IR calorimetry and by water calorimetry [15] is shown in figure 5 for about five thousand pulses, both in hydrogen and deuterium. A linear fit of the whole data set is performed: the very good agreement between the two measurements allows the estimation of $j_{\text {acc }}$ retrieved by IR calorimetry to be validated. The linear fit is not forced to zero to check for the presence of an offset between the two measurements: the offset is $2.1 \mathrm{~A} / \mathrm{m}^{2}$, which is very small compared to the typical value measured $(\leq 5 \%)$. By considering the error bars of both diagnostics, i.e. $\pm 5 \%$ of the IR calorimetry and $\pm 10 \%$ of the water calorimetry, the offset is compatible with the measurement error. The goodness of the fit is given by Pearson's $r$ coefficient and it is very good in this case, being the value close to one. For the averaged accelerated current $\mathrm{j}_{\text {acc }}$ over the calorimeter surface, IR calorimetry and water calorimetry are equivalent but, as presented in the next section, IR calorimetry is preferable because it gives a spatial resolution of $4 \mathrm{~cm}$. 


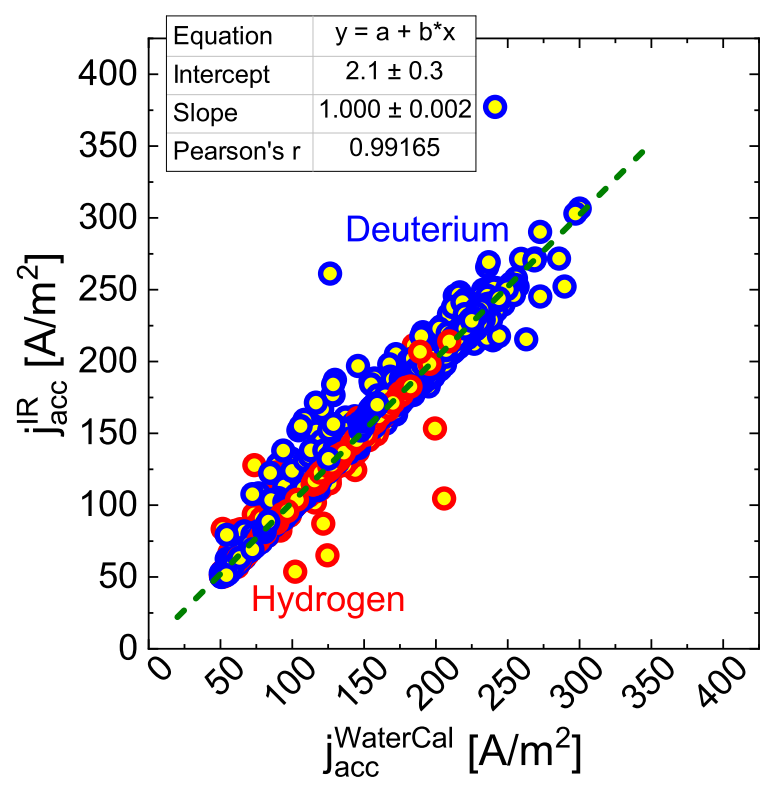

Figure 5: Comparison between the accelerated current density $\mathrm{j}_{\mathrm{acc}}$ as measured by water calorimetry and by IR calorimetry. The pulses are done between January 2017 and July 2018, both in hydrogen and deuterium. A linear fit is applied to the whole data set: the resulting slope is $1.000 \pm 0.002$ and the intercept is $2.1 \pm 0.3$. The goodness of the fit is given by the Pearson's coefficient. Few cases are outside of the main cloud of data: this is due to an error on the automatic evaluation of the effective emissivity which can not be detected a priori but only at a later time by comparing the value with the water calorimetry measurement.

1

\subsection{Fitting procedure}

The 2D map of the beam power deposited onto the calorimeter provides a qualitative insight on the beam profile: a fitting routine is applied in order to retrieve quantitative information on the beamlet group vertical and horizontal width, intensity and on the vertical position of the beam segments. The fitting formula is a sum of eight bidimensional Gaussian functions that represent the eight rectangular beamlet groups. The Gaussian shape assigned to the power density profile was chosen starting from the hypothesis that at high distance from the grid system the overlap of beamlets arranged as the apertures in figure 6(a), gives a Gaussian profile [23]. In equation 4 the fitting function as a function of the vertical and horizontal coordinates ( $\mathrm{y}$ and $\mathrm{x}$ in the equation, respectively) is given. The top and bottom beam segments are treated separately: the subscripts "top" and "bot" refer to the top and bottom beam segment, respectively. The schematic representation of the fitting function superimposed to a power density profile is shown in figure $6(\mathrm{~b})$.

$$
f(x, y)=\sum_{i=1}^{4} A_{i} e^{-\frac{\left(x-x_{0, i}\right)^{2}}{2\left(\sigma_{x}^{\text {top }}\right)^{2}}-\frac{\left(y-y_{\text {top }}\right)^{2}}{2\left(\sigma_{y}^{\text {top }}\right)^{2}}}+\sum_{i=5}^{8} A_{i} e^{-\frac{\left(x-x_{0, i}\right)^{2}}{2\left(\sigma_{x}^{\text {bot }}\right)^{2}}-\frac{\left(y-y_{\text {bot }}\right)^{2}}{2\left(\sigma_{y}^{\text {bot }}\right)^{2}}}
$$

The horizontal position of the Gaussians $x_{0, i}$ is fixed and it corresponds to the geometrical center of the beamlet groups on the GG. The vertical positions of the top and bottom beam segments $\left(y_{\text {top }}\right.$ and $\left.y_{\text {bot }}\right)$ are free parameters due to the vertical 


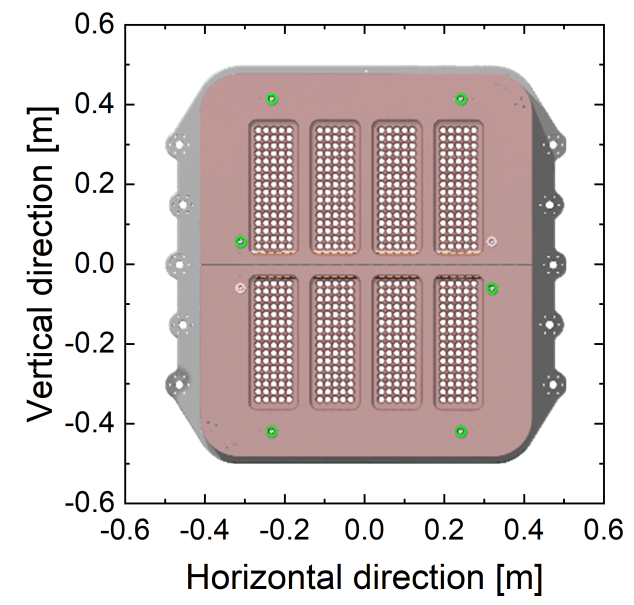

(a)

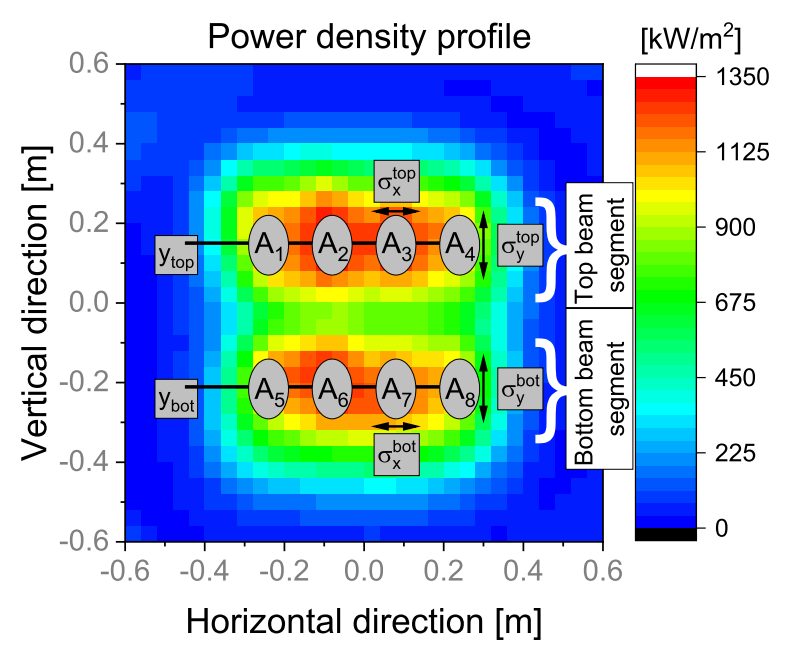

(b)

Figure 6: (a) Aperture arrangement on the PG. (b) Schematic of the fitting function applied to the 2D map of the power density on the calorimeter. The parameters of the fitting function are superimposed to power density profile. The top and bottom beam segments enclose the beamlet groups located on the top and on the bottom side of the calorimeter, respectively.

deflection of the beam caused by the filter field. The vertical and horizontal width $\left(\sigma_{y}\right.$ and $\sigma_{x}$ ) of the Gaussian are assumed identical for the four beamlet groups in each beam segment because no inhomogeneity among beamlet groups belonging to the same beam segment is expected. The amplitude of each Gaussian function is described by the free parameters $A_{i}$, with $i=1 \ldots 8$. The error bar assigned to the fit outputs $\sigma_{x}, \sigma_{y}, y_{\text {top }}$ and $y_{\text {bot }}$ is calculated from the error on the fitting procedure and from the error due to the dimension of the calorimeter block. The error on the position due to the dimension block is calculated as $\frac{3.8 \mathrm{~cm}}{\sqrt{12}}=1.1 \mathrm{~cm}$, where $3.8 \mathrm{~cm}$ is the dimension of the block and $\sqrt{12}$ is the factor to obtain the standard deviation from the width of the uniform distribution considered [24], namely the block width in this case. The error due to the dimension of the block is the dominant contribution to the error on the fit outputs.

In this model, the integral of the the Gaussian function describing one beamlet group

is proportional to the accelerated current carried by the beamlets belonging to that beamlet group. The integral is calculated by integrating each Gaussian function to infinity, thus including beam losses outside the calorimeter surface $(\leq 10 \%$ or negligible in the majority of the cases) which can occur in case of high beam divergence or high beam deflection caused by a strong filter field. The accelerated current can be calculated for each beamlet group and in the case of the top beam segment is

$$
V_{\mathrm{top}}^{\mathrm{fit}}=\int_{-\infty}^{+\infty}\left(\sum_{i=1}^{4} A_{i} e^{-\frac{\left(x-x_{0, i}\right)^{2}}{2\left(\sigma_{x}^{\mathrm{top}}\right)^{2}}-\frac{\left(y-y_{\mathrm{top}}\right)^{2}}{2\left(\sigma_{y}^{\mathrm{top}}\right)^{2}}}\right) d x d y=2 \pi \sum_{i=1}^{4} A_{i} \sigma_{x}^{\mathrm{top}} \sigma_{y}^{\text {top }}
$$

and the corresponding case for the bottom is $V_{\text {bot }}^{\text {fit }}=2 \pi \sum_{i=5}^{8} A_{i} \sigma_{x}^{\text {bot }} \sigma_{y}^{\text {bot }}$.

In order to test the eligibility of the fit, it has been applied to synthetic power profiles. 
The synthetic profiles are produced by assuming a Gaussian power distribution for each of the 640 beamlets at the exit of the GG and projecting them up to the calorimeter distance. No beamlet deflection is foreseen. At the calorimeter each beamlet has a Gaussian profile and the width $\delta$ is calculated as

$$
\delta=\frac{1}{\sqrt{2}} 3.5 m \tan \left(\vartheta_{\frac{1}{e}} \cdot \frac{\pi}{180}\right),
$$

where $\vartheta_{1 / e}$ is the divergence in degrees at one e-folding half width and $3.5 \mathrm{~m}$ is the distance between GG and diagnostic calorimeter. The factor $\frac{1}{\sqrt{2}}$ is the conversion factor between the width in sigma and the e-folding half width. For every aperture a Gaussian function $p_{i}(x, y)$ is used. The total power on the calorimeter $p(x, y)$ is

$$
p(x, y)=\sum_{i=1}^{640} p_{i}(x, y)=\sum_{i=1}^{640} \frac{a_{i}}{2 \pi \delta_{x} \delta_{y}} e^{\frac{-\left(x_{i}-x\right)^{2}}{2 \delta_{x}^{2}}-\frac{\left(y_{i}-y\right)^{2}}{2 \delta_{y}^{2}}},
$$

where $a_{i}$ is the peak amplitude of the single Gaussian function describing the beamlet, $x_{i}$ and $y_{i}$ are the horizontal and vertical beamlet coordinates defined in the reference system of figure $6(\mathrm{a})$, and $\delta_{x}$ and $\delta_{y}$ are the horizontal and vertical beamlet width. The following assumptions have been used:

- All the beamlets are circular $\left(\delta_{x}=\delta_{y}\right)$;

- The peak intensity $a_{i}$ is the same for beamlets belonging to the same beamlet group;

- The divergence is assumed to be the same for apertures belonging to the same beam segment (top or bottom);

The validity of these assumptions are discussed in section 4.3 with a comparison between simulation and a real pulse performed at ELISE. Fluctuations are simulated by adding a random noise of $\pm 10 \%$ of the absolute values (uniform distribution). The resolution of the synthetic profile has been reduced to mimic the experimental resolution of the blocks on the calorimeter.

Once the synthetic profiles are produced and the fit is applied, it is possible to correlate the behavior of the output parameters with the simulation ones, such as the dependence between beamlet divergence $\vartheta$ and beamlet group width $\sigma_{x}$ and $\sigma_{y}$ as shown in figure $7(\mathrm{a}): \sigma_{x}$ and $\sigma_{y}$ for the top and bottom beam segments obtained from the fitting routine are plotted as a function of $\vartheta$. In each simulation shown in figure $7(\mathrm{a})$, the intensity and the divergence are the same for all beamlets, thus the beam is horizontally and vertically symmetric with respect to the zero of the scale. The divergence varies from $\vartheta=1$ degree to $\vartheta=5$ degrees in steps of 0.2 degrees. While increasing the beamlet divergence, the width of the beamlet groups in the power density profile increases. The behavior of $\sigma_{y}$ is linear above a divergence of about 2.5 degrees, below it $\sigma_{y}$ still decreases with decreasing divergence but not linearly. The beam vertical width is determined by the divergence and only for small values of divergence by the geometrical arrangement of the apertures on the grids. The horizontal width $\sigma_{x}$ always increases linearly with increasing divergence. In figure 7(a) on the right axis the adjusted R-square of the fit as a function of $\vartheta$ is shown. The adjusted R-squared is an estimator of the goodness of the 
(a)

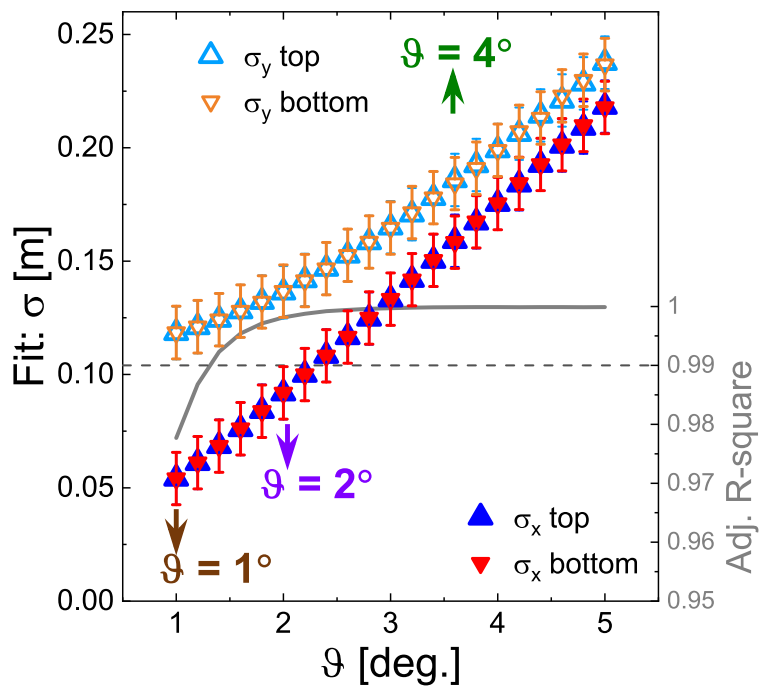

(b)
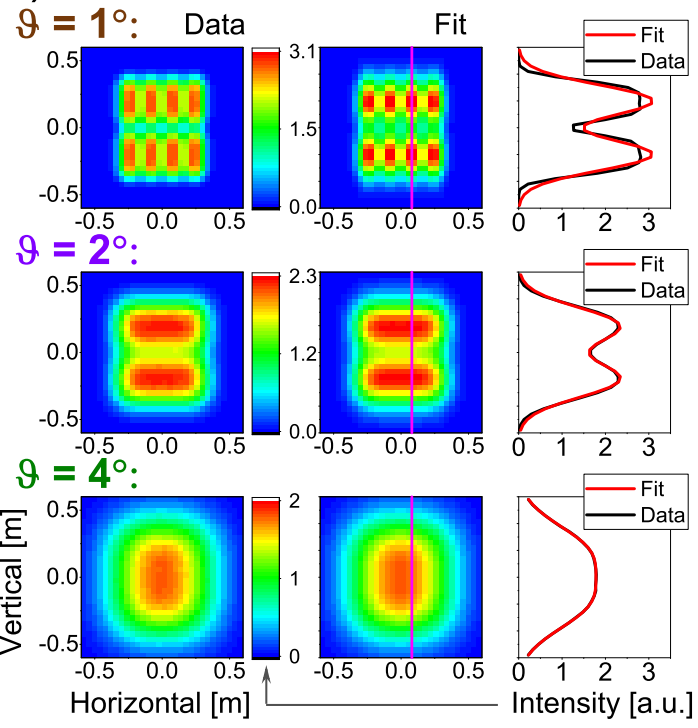

Figure 7: (a) Vertical and horizontal beamlet group width calculated by the fitting routine as a function of the divergence used in the synthetic 2D map; the intensity is the same for all the beamlets. For the cases with 1,2 and 4 degree divergence a plot of the synthetic data, of the fitted matrix and of the vertical profile is shown in panel (b). In (a), on the right axis, the adjusted R-square calculated is shown: the level of 0.99 indicates the minimum level accepted, below 0.99 the simulated map is not properly reproduced by the fitting routine.

fit and the threshold used to accept the result is 0.99 . Below it, the fitting function is not representative of the $2 \mathrm{D}$ profile. The lowest value of beamlet divergence $\vartheta$ for which the fitting function properly reproduces the $2 \mathrm{D}$ map is 1.5 degrees.

In figure $7(\mathrm{~b})$, the $2 \mathrm{D}$ maps and profiles from simulations with 1,2 and 4 degrees divergence and the corresponding fits are shown: the synthetic 2D maps is shown in the first column, the fitted one is shown in the second column and in the third column the vertical profiles from the synthetic map and from the fitted one are displayed. The overlap of Gaussian beamlets at the calorimeter plane gives a beamlet group profile that is not, in general, a Gaussian, especially for low divergences as shown in the figure 7(b) for the case at 1 degree: in this case, the beamlet group profile becomes flat in the central part, resulting in a rectangle rather than a bell-shaped curve. At 1 degree divergence (17.4 mrad which is close to the calculated optimum of $14.4 \mathrm{mrad}-19.5 \mathrm{mrad}$ for ELISE[12]), the fitting procedure does not properly reproduce the synthetic map: the intensity (i.e. $A_{i}$ in equation 4 ) is overestimated and the beam vertical width $\sigma_{y}$ is underestimated. A different type of fitting function, e.g. a box function or a flat-top Gaussian curve, can also be applied for the cases with divergence lower than 1.5 degrees, but in such cases the number of points is not sufficient for a robust and reliable automatic evaluation of the IR 2D profile. The agreement is good at higher divergence as shown for 2 and 4 degrees divergence in figure 7 (b). At 4 degrees, where the beam segments are indistinguishable because of the large overlap, the fitting routine reproduces the power 
1

profile correctly.

Up to this point, the test is performed on a homogeneous beam, i.e. all the beamlets have the same divergence and intensity. In the following, the fitting routine is tested on a larger set of simulations, where asymmetries of the beamlet divergence and beamlet intensity between beam segments, but also among beamlet groups, are introduced.

4.2.1. Beam width The fitting routine is tested on the capability to characterize the two beam segments independently from one other. The test is carried with a set of simulations in which the inputs of the top beam segment are fixed while the bottom ones vary. If the fit outputs for the two beam segments are independent on from each other, the test is then successful.

In figure 8 some significant cases are displayed: dots correspond to the simulated profile, lines to the fit. In each panel $\vartheta_{\text {top }}$ is fixed and $\vartheta_{\text {bot }}$ varies according to the values shown in each panel. In panels (a) and (b) the beamlets in the simulation have the same intensity between top and bottom beam segment while in (c) and (d) the bottom beamlets are three times more intense than the top ones. Such large variations on the input parameters represent scenarios with high inhomogeneity in the current density and in the beam divergence between beam segments. In each panel, the fit correctly reproduces the beam profile in all the cases and $\sigma_{\mathrm{y}}^{\text {top }}$ coincide within the error bars as shown by the overlapping of the black curves. Larger differences on the vertical width $\sigma_{\mathrm{y}}^{\text {top }}$, but always within the error bars, are observed in (c) and (d) due to the large difference $(3 \times)$ in intensity between the top and bottom beam segments.

4.2.2. Beam integral The proportionality between beam segment integral in the simulations and calculated from the fit results has been checked under different conditions. This test is fundamental for the correct evaluation of the accelerated current for each beamlet group or beam segment. The beam integral calculated from fit routine outputs (see equation 5) is $V_{\mathrm{top}}^{\mathrm{fit}}=2 \pi \sum_{i=1}^{4} A_{i} \sigma_{\mathrm{x}}^{\text {top }} \sigma_{\mathrm{y}}^{\text {top }}$ and the corresponding case for the bottom is $V_{\mathrm{bot}}^{\mathrm{fit}}=2 \pi \sum_{i=5}^{8} A_{i} \sigma_{\mathrm{x}}^{\text {bot }} \sigma_{\mathrm{y}}^{\text {bot }}$. For the simulation, the top beam segment integral is calculated as

$$
V_{\text {top }}^{\text {sim }}=\int_{-\infty}^{+\infty}\left(\sum_{i \in \text { top }} \frac{a_{i}}{2 \pi \delta_{\mathrm{x}} \delta_{\mathrm{y}}} e^{\frac{-\left(\mathrm{x}_{i}-\mathrm{x}\right)^{2}}{2 \delta_{\mathrm{x}}^{2}}-\frac{\left(\mathrm{y}_{i}-\mathrm{y}\right)^{2}}{2 \delta_{\mathrm{y}}^{2}}}\right) d x d y=\sum_{i \in \text { top }} a_{i}
$$

and the corresponding case for the bottom is $V_{\text {bot }}^{\text {sim }}=\sum_{i \in \text { bot }} a_{i}$. In figure 9 the quantity $\left(V_{\text {top }}-V_{\text {bot }}\right) / V$ where $V$ is the global integral, calculated from the fit outputs as a function of the one retrieved from the simulation inputs is shown. In the simulations, the bottom beam segment intensity is varied while the top one is fixed. In (a) $\vartheta_{\text {top }}=\vartheta_{\text {bot }}$, in (b) $\vartheta_{\text {bot }}=2$ degrees and in (c) $\vartheta_{\text {bot }}=4$ degrees. Additionally, $\vartheta_{\text {top }}$ is varied and the values are shown in each panel of figure 9. The two ratios (of the fitted and of the synthetic profiles) are proportional and all within $10 \%$ of the bisector shown as a solid line in each panel and the shaded area around the bisector represents 

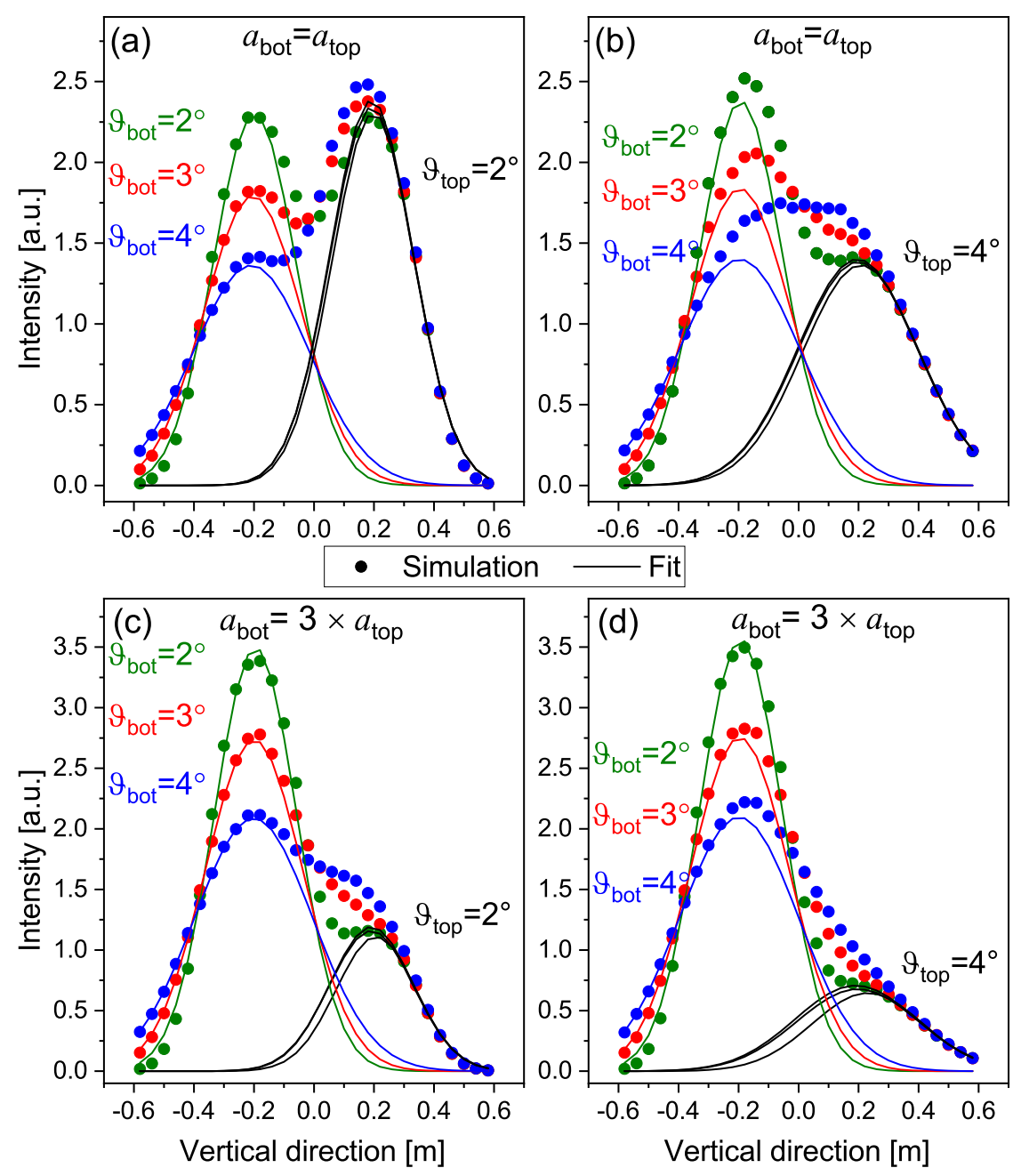

Figure 8: Simulated vertical profiles (dots) for different cases and fitted profiles (lines). The fitted profiles for the top and bottom segments are plotted separately. In each panel the top beamlet divergence is constant while the bottom one is set at 2,3 and 4 degrees. The top divergence is fixed to 2 degree in (a) and (c) and to 4 degrees in (b) and (d). In panel (a) and (b) the beamlets in the simulated top and bottom beam segments have the same peak intensity while in (c) and (d) the beamlets in the bottom segment are three times more intense that the top ones.

$\pm 10 \%$ deviation. Thus, the accelerated current carried by each beam segment is reliably estimated from the fit outputs. Similar tests have been done successfully for varying the relative intensity of single beamlet groups: the beamlet group integral is always 4 proportional to the simulated values, confirming the capability of the fitting routine to 5 correctly estimate the intensity of each beamlet group and, in turn, of the beam segment.

7 4.2.3. Beam position The vertical positions of the top and bottom beam segment are 8 free parameters of the fitting routine due to the beam vertical deflection. To simulate 9 the vertical deflection, the apertures on the grids are vertically displaced keeping the 


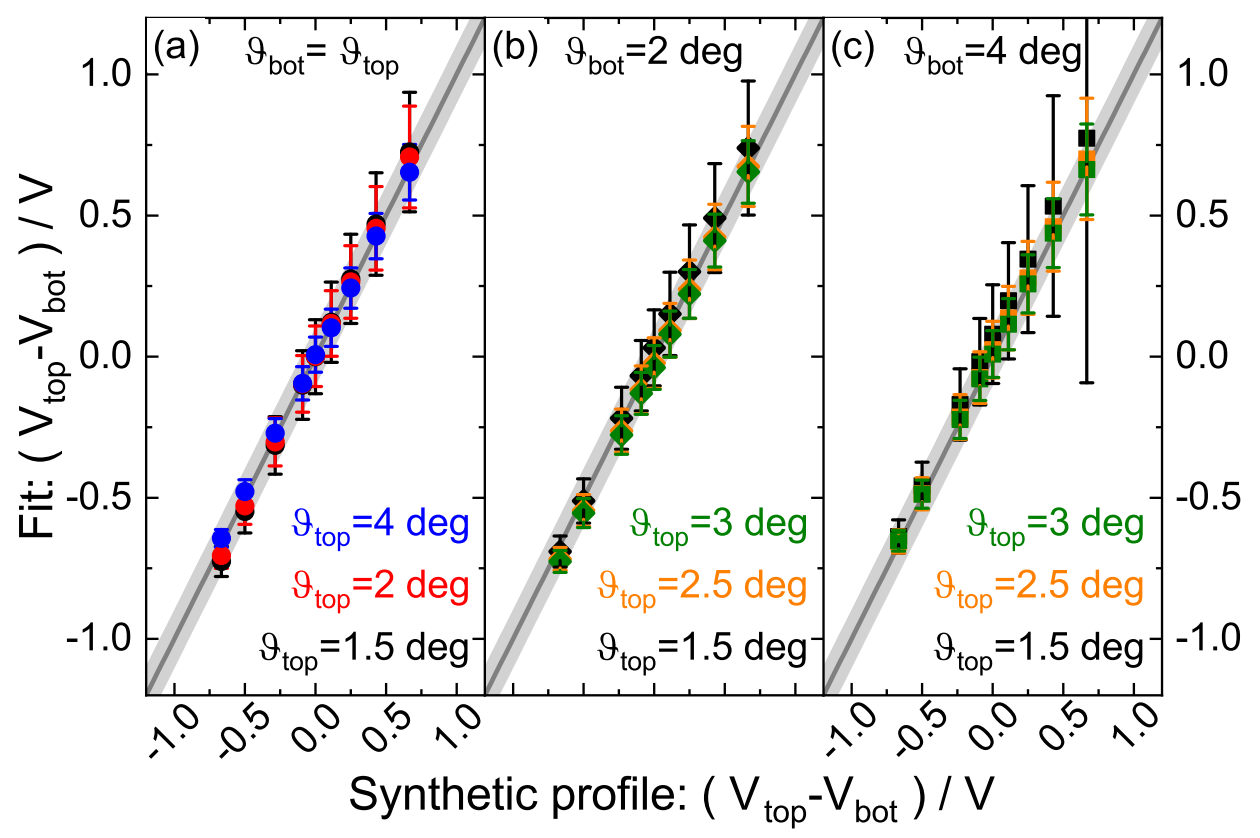

Figure 9: Proportionality between simulation and fit on the ratio of the beam segment integrals. The bottom beamlet intensity was varied with respect to the top one. Three cases are shown: when $\vartheta_{\text {top }}=\vartheta_{\text {bot }}$ in (a), when $\vartheta_{\text {bot }}=2$ degrees in (b) and when $\vartheta_{\text {bot }}=4$ degrees in (c). The solid lines identify the bisector in each plot.

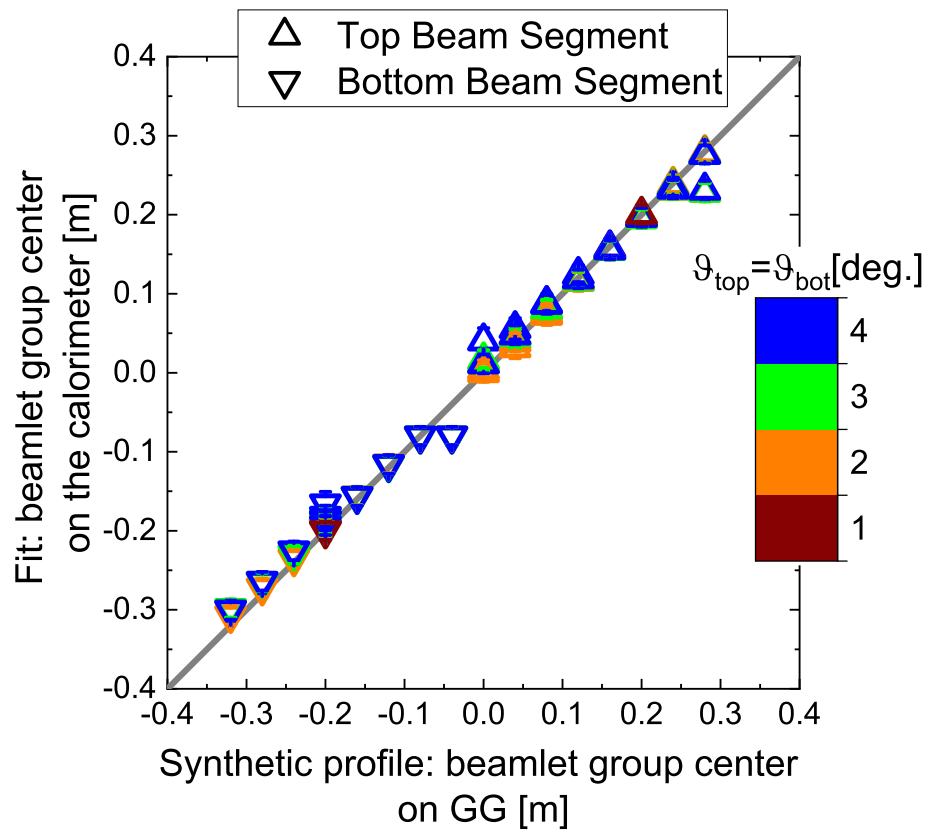

Figure 10: Beam vertical position retrieved by the fit as a function of the beamlet group center position at the GG and for different divergences $\left(\vartheta_{\text {top }}=\vartheta_{\text {bot }}\right)$ according to the color scale. The solid line identifies the bisector. 
beamlet group structure. Two different sets of cases have been tested:

- the position of one beam segment is fixed, the bottom one in this case, while the other one is varying. This case mimics a scenario in which variations of the vertical beam segment homogeneity are detected by variation of $\sigma_{y}$ and vertical position. In this case the two beam segments can react independently.

- the position of both segments is displaced vertically of the same quantity. These cases concern the scenario of different filter field intensity or different HV applied, thus affecting the vertical displacement of both segments in the same way.

The fit outputs $y_{\text {top }}$ and $y_{\text {bot }}$ are shown in figure 10 as a function of the beamlet group center position used for the simulation. Beamlet divergences between $1^{\circ}$ and $5^{\circ}$ in steps of $0.5^{\circ}$ have been considered and some cases are reported in figure. The agreement between the fit and the simulation is very good, also at four degrees divergence when it is qualitatively difficult to distinguish the two beam segments at the calorimeter due to the large overlap as shown in figure 7 for the case at four degrees divergence.

\subsection{Comparison of simulations with an experimental beam pulse}

The 2D power density map of the beam shown in figure 3(c) is compared to a simulation in order to check if the assumptions of the simulation are reasonable, especially regarding the uniformity in intensity for all the beamlets belonging to one beam segment. The input values for the simulation are:

- $\vartheta_{\text {top }}=\vartheta_{\text {bot }}=1.8$ degrees determined by BES for the horizontal LOS looking at the center of each beam segments (in this case the value is 1.8 degrees for both beam segments).

- $2.5 \mathrm{~cm}$ of vertical displacement downwards taken from the fit on the IR data: the value is $2.5 \mathrm{~cm}$ downwards for the top beam segment and $2.9 \mathrm{~cm}$ downwards for the bottom beam segment, the value are in agreement within the error bar. For simplicity, only $2.5 \mathrm{~cm}$ is chosen.

- All the beamlets have the same peak intensity since the vertical profile measured by IR calorimetry shows that the two beam segments have a similar intensity (see BES intensity profile in figure 2(b) and 2D map in figure 3(c)).

The power density map for the experimental beam pulse and the simulation are shown in figure 11(a) and (b), respectively. In the experimental map, as well as in the simulated one, the beam segments are clearly detached from one another. The inner beamlet groups in the experimental case, especially in the top beam segment, can be distinguished while in the simulation they are barely distinguishable.

A more quantitative comparison is carried out by comparing the vertical and horizontal profiles in figure 11(c) and (d), respectively. The vertical profiles are plotted at two positions illustrated in figure 11(a) and (b) with the label "Outer B.G." and "Inner B.G." for the outer and inner beamlet groups, respectively. 


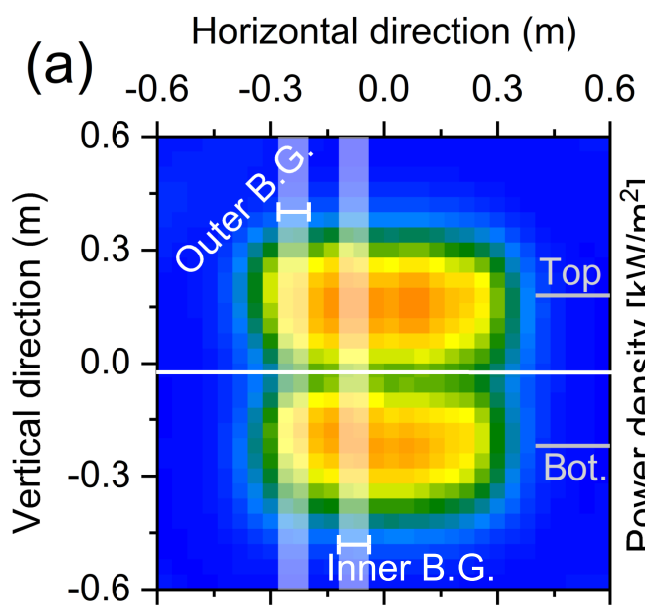

(c)

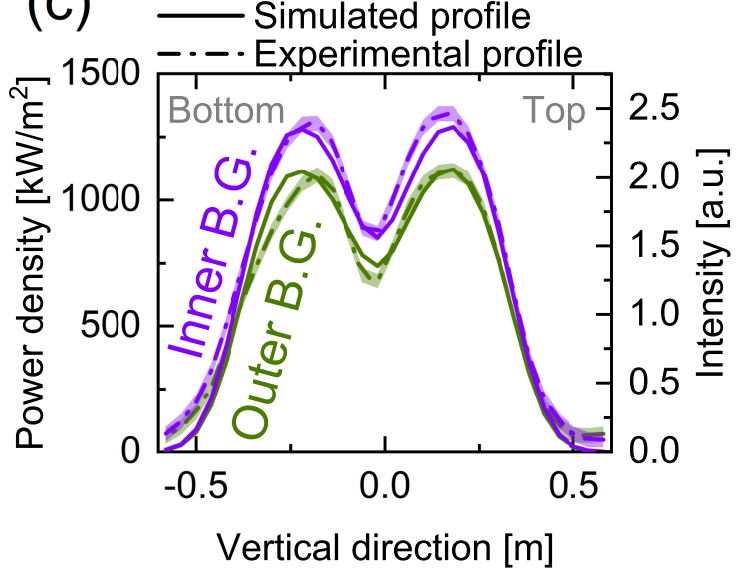

(b) Horizontal direction [m]

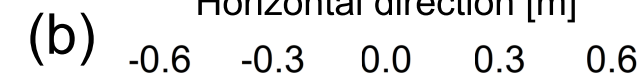

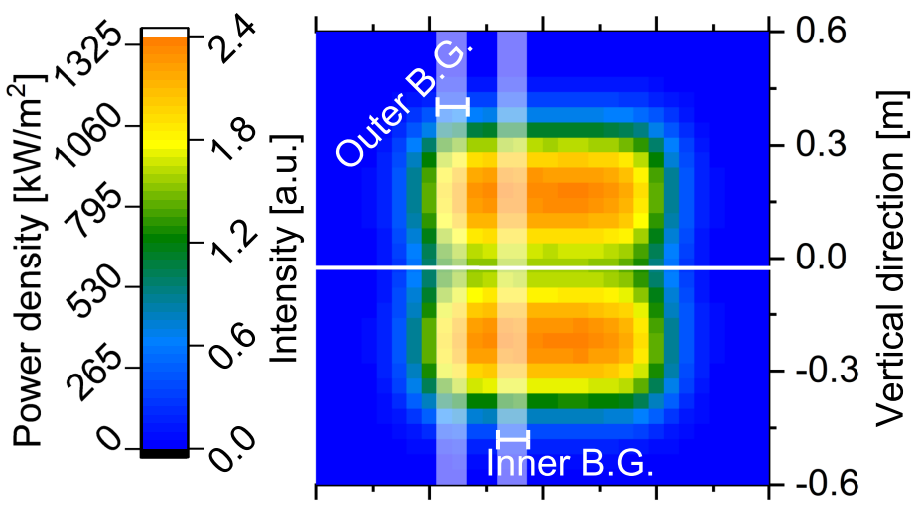

(d)

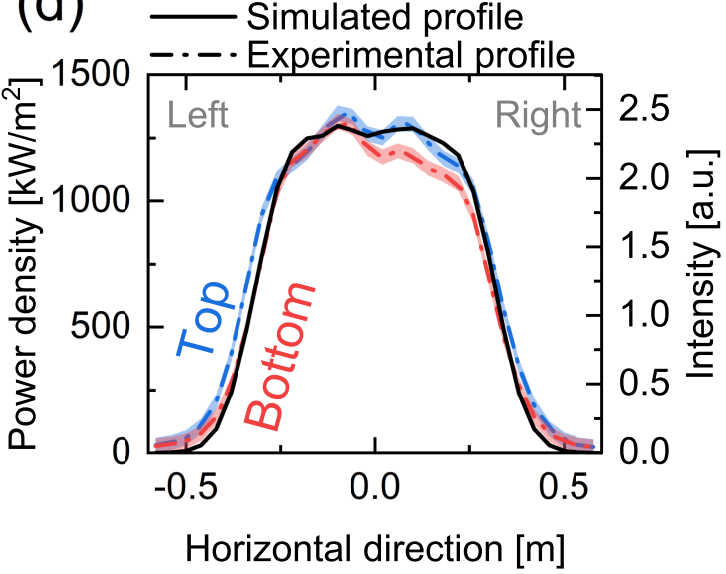

Figure 11: (a) 2D power density map for one ELISE beam pulse, to be compared with the simulated 2D map (input parameters: $\vartheta_{\text {top }}=\vartheta_{\text {bot }}=1.8^{\circ}$, uniform intensity for all the beamlets, shift of $2.5 \mathrm{~cm}$ downwards retrieved by the fit) shown in (b). The solid white horizontal line depicts the beam center calculated by the IR fit applied to (a) and used as parameter in (b). In panel (c) two measured vertical profiles are compared with the simulation: the vertical profiles are given for the outer beamlet groups (Outer B.G.) and the inner beamlet groups (Inner B.G.) i.e. the leftmost beamlet groups and the second beamlet groups from the left, respectively. In panel (d) the horizontal profiles of the top and bottom beam segments are compared with the simulation: the positions for the comparison are shown in panel (a). The shaded area around the experimental profiles in (c) and (d) is the experimental uncertainty on the power density. In (c) and (d) the experimental profiles refer to the left axis while the simulations to the right axis.

1 The horizontal profiles are plotted along the center of the top and bottom beam segment as shown in figure 11(a) by the levels labeled with "Top." and "Bot.". The vertical profiles are plotted in figure 11(c) for the inner and outer beamlet groups. The inner beamlet groups are more intense than the external ones due to the beamlet group overlapping that increase the intensity in the center. The shaded area around the experimental profiles in fig 11(c) and (d) is the experimental uncertainty on the power density.

The simulation reproduces quite accurately the vertical profile in particular for the top beam segment and with minor deviations localized on the bottom beam segment: 
the maximum of the bottom beam segment is located in a different position and the intensity profile is different. This discrepancy may indicate that the assumption that all the beamlets within one segment have the same intensity is not appropriate, especially for the bottom beam segment where a more appropriate assumption would be a higher intensity for the upper rows of beamlets and a lower intensity in the remaining ones. The experimental horizontal profile in figure 11(d) shows that the total beam width is comparable to the one simulated considering the error bars. The beamlet groups are more distinguishable in the experimental profile, thus suggesting that the real beamlet divergence is lower than the one measured by BES and used as input for the simulation probably due to the left-right beamlet deflection. A priori, the assumption that all the beamlets carry the same extracted current cannot be verified, but the comparison of the vertical and horizontal profiles between values measured by IR calorimetry and a simulation as shown in figure 11(c) and (d) suggests that it is reasonable in a first approximation.

\section{Crosscheck of IR calorimetry with BES diagnostic}

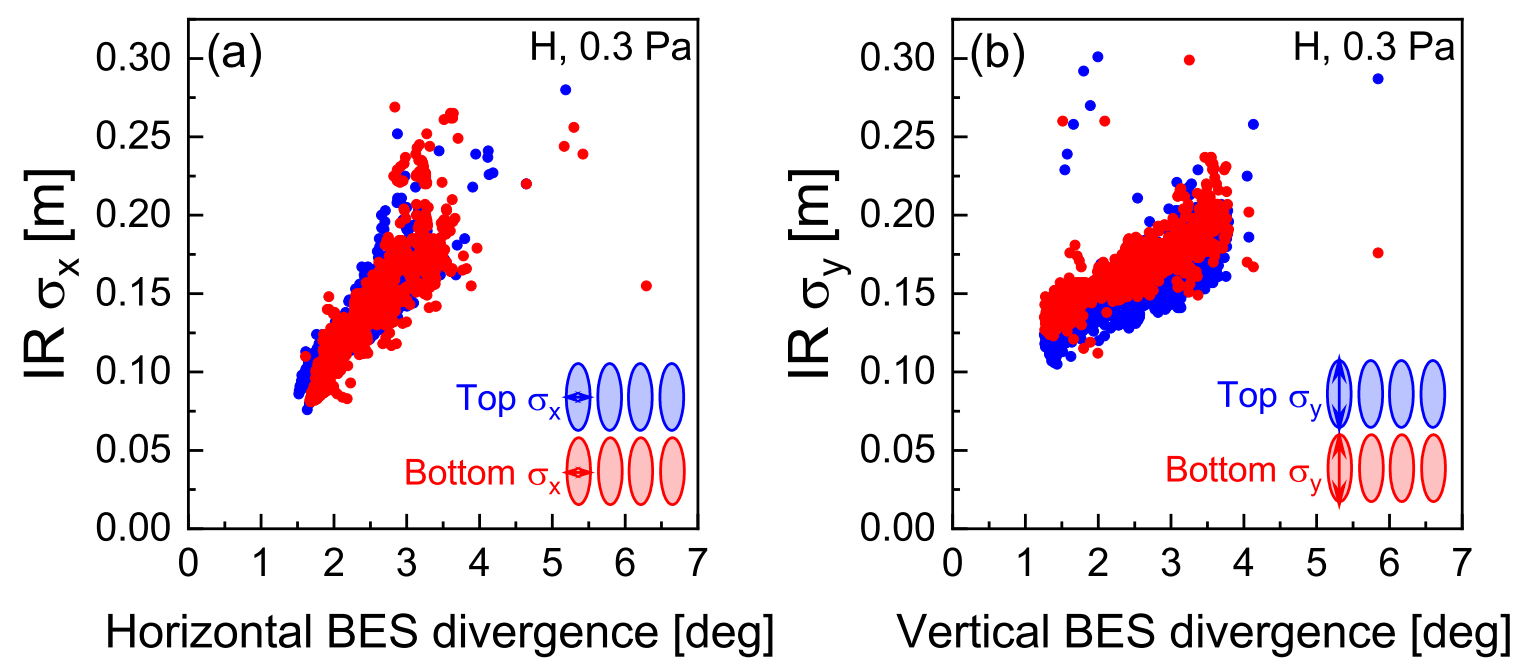

Figure 12: Comparison between IR calorimetry and BES. In (a) the horizontal width $\sigma_{\mathrm{x}}$ is plotted as a function of the BES horizontal divergence. In (b) the vertical width $\sigma_{\mathrm{y}}$ is plotted as a function of the BES vertical divergence. About 4000 pulses performed in hydrogen at 0.3 Pa between January 2017 and July 2018.

Around 4000 hydrogen beam pulses performed at the $0.3 \mathrm{~Pa}$ are used to crosscheck the IR calorimetry technique with the BES diagnostics, particularly regarding the relation between beam width and BES divergence. The beam segment width $\sigma$ from IR calorimetry as a function of the beam divergence from BES is displayed in figure 12 , separately for the horizontal $\sigma_{x}$ in (a) and the vertical $\sigma_{y}$ in (b). The horizontal divergence from BES is the average of the divergence measured by three LOS looking at the center of the top and bottom beam segments while the vertical divergence is an 
average of the values obtained from the two central vertical LOS. The vertical BES LOS measure the whole beam, so it is not possible to distinguish between the contribution from the top and the bottom segments.

In both directions, there is good overall agreement between the beam width estimated by IR calorimetry and the beam divergence from BES, thus the beam width is, to a large extent, determined by the beam divergence. Nevertheless, the data points are scattered in a range of about $5 \mathrm{~cm}$ for the same beam divergence: this can be explained by a vertical non-homogeneity in beamlet intensity ( $a_{i}$ of equation 7 ) within the beamlet groups. The bottom vertical width $\sigma_{\mathrm{y}}$ (red points in figure $12(\mathrm{~b})$ ) is systematically larger of about $2 \mathrm{~cm}$ than the top one for the same BES divergence, thus not within the error bar. This is a general effect and the reason is attributed to the vertical inhomogeneity of the source plasma parameters.

The combined use of BES diagnostics and IR calorimetry gives additional insight into the beam properties: BES is sensitive to variations of divergence without any spatial resolution along the LOS, while IR calorimetry gives the $2 \mathrm{D}$ power map at a fixed position with spatial resolution but without information on the divergence. Thus a variation in power density cannot be directly attributed to a different divergence or different power homogeneity.

In the following example, the concurrent use of the two diagnostics is applied to study the evolution of the beam power homogeneity during an RF power variation. In figure 13 the vertical power density profile from IR calorimetry (bottom), beam intensity profile measured by BES (center) and beam divergence profile from BES (top) are shown. The beam intensity profiles given by BES (figure 13 center) and by IR calorimetry (figure 13 bottom) show a similar evolution with the RF power and the divergence profile is in general flatter at higher RF power. The behavior of the beam segments is described in the following:

- The top beam segment saturates in intensity and the beam segment width increases with power from $0.117 \mathrm{~cm}$ to $0.128 \mathrm{~cm}$. This increase of the vertical width is not caused by an increase in the beam divergence, which is only slightly varying (see figure 13 top), but due to flattening of the beam intensity profile. BES measurements together with IR calorimetry suggest that the vertical power profile gets more homogeneous with increasing RF power, as the extracted current within the beam segment is more homogeneous at $240 \mathrm{~kW}$ than at $160 \mathrm{~kW}$.

- The bottom beam segment shows a different evolution with increasing RF power: the intensity increases (see figure 13 center and bottom) and the beam divergence slightly improves from $0.146 \mathrm{~m}$ to $0.12 \mathrm{~m}$ with the RF power (see figure 13 top). In this case the vertical width decreases because the beam divergence improves with RF power. The beam segment intensity increases with RF power being the beamlets more focused and the extracted current higher. The vertical beam width follows the variation of the beam divergence thus suggesting that the vertical homogeneity of the bottom beam segment is not changed. 


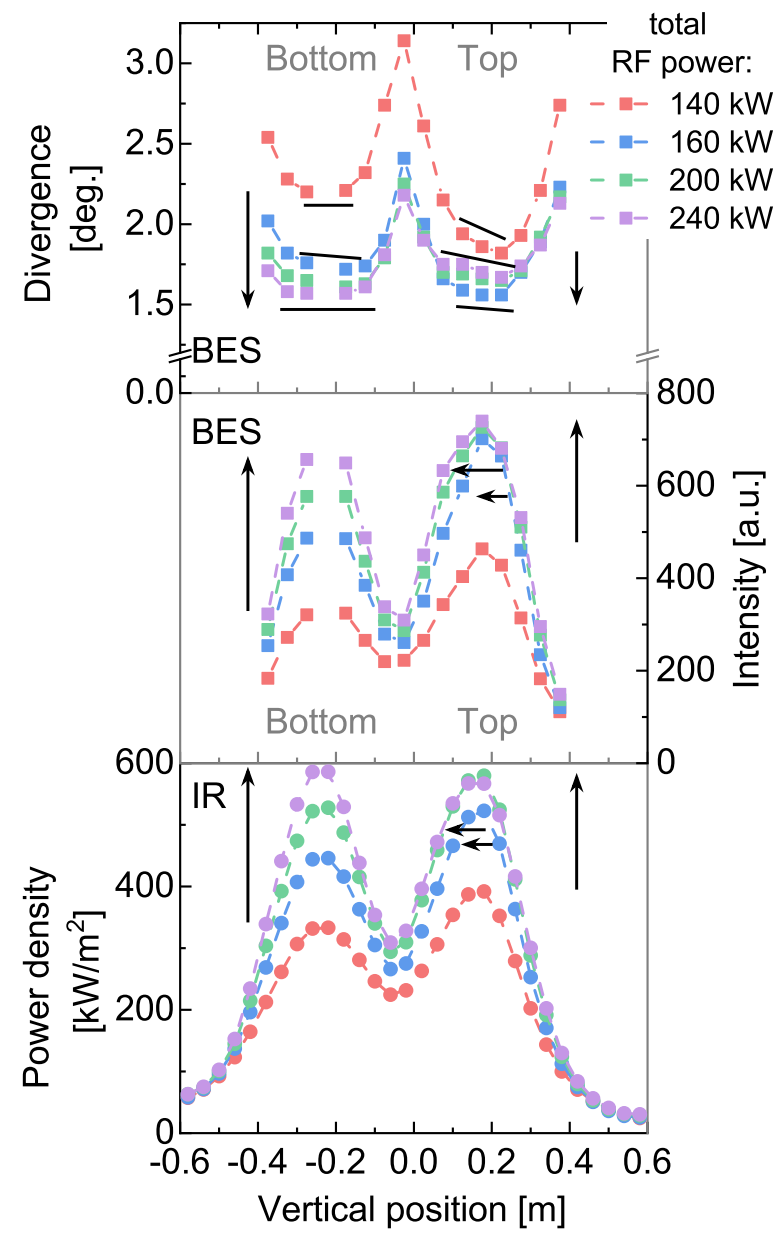

Figure 13: RF power scan performed in deuterium at 0.3 Pa source filling pressure, shot 22617-22624 in the range between $140 \mathrm{~kW}$ and $240 \mathrm{~kW} R F$ power, $\mathrm{U}_{\text {ext }}=6 \mathrm{kV}, \mathrm{U}_{\text {acc }}=30 \mathrm{kV}$ and $I_{P G}=3.7 \mathrm{kA}$. In (a) the vertical beam profile for the divergence (top), beam intensity measured by BES (center) and power profile measured by IR calorimetry (bottom) are shown. The LOS positioned at $22.5 \mathrm{~cm}$ is not available. The evolution of the beam divergence and intensity profiles is highlighted with purple arrows. 


\section{Conclusions}

IR calorimetry and BES diagnostics applied to the large multi-aperture beam at ELISE provide measurements of the beam divergence and accelerated current with an accuracy of $10 \%$ on the measured values. The IR calorimetry evaluation technique is developed, described and verified, thus providing measurements of the beamlet group width, beamlet group power density, and vertical beam position with an accuracy of $1.2 \mathrm{~cm}$ on the position and $10 \%$ on the power density. Due to the large beamlet overlap occurring at the diagnostic planes, the beam properties are averaged over groups of beamlet, thus not representative of the single beamlets.

The calibration procedure applied to determine the effective surface emissivity of the calorimeter is accurate, i.e. 5\% error bar, and reliable as demonstrated by the crosscheck of the IR calorimetry results with water calorimetry. A fitting routine which describes the beamlet group profile as Gaussian is applied to the 2D map obtained from the IR calorimetry to retrieve the beamlet group width, beamlet group intensity, vertical beam position and accelerated current. The fit properly describes cases with a simulated beamlet divergence higher than about 1.5 degrees. For cases with lower simulated beamlet divergence the beamlet group profile is not Gaussian, therefore the fitting routine is not applicable. The fitting routine correctly reproduces the power density profile of cases with strong asymmetries between beam segments independently of the beam divergence, thus is a valuable tool to retrieve the beam properties from the IR $2 \mathrm{D}$ map.

Results from IR calorimetry and BES are compared over a large set of experimental beam pulses to verify that the beamlet group width is proportional to the beam divergence, thus the beam width at the calorimeter is determined mainly by the beam divergence for cases with BES divergence higher than 1 degree. For certain parameter variations, IR calorimetry and BES diagnostic have to be applied jointly to assess properly if a variation of the power density observed at the calorimeter is due to a change in divergence or a change in power density homogeneity.

To reduce the effect of the beamlet overlapping on the BES results, the LOS should be positioned closer to the grid system and with a smaller volume of observation of the lens head. However this will make the analysis more difficult due to the partial interception of the beamlets with the LOS. A larger number of LOS can improve the spatial resolution. For large beams where beamlet overlapping is inevitable an increase in diagnostic precision will still not be able to give beamlet level information. Therefore, coupling diagnostic measurements with simulation code such as IBSIMU [11] and BBCNI [17] is necessary for a better insight of the single beamlet properties.

On the side of the diagnostic calorimeter a smaller block size would be beneficial for the spatial resolution, but will still not allow single beamlet measurements. Furthermore, having the diagnostic calorimeter closer to the grid will only increase the power density deposited locally and the risk of localized damages on the surface. For the calorimetric study of single beamlets other tools should be used, with the drawback of limiting the 
power deposition and/or the beam-on time. An example of such tools are tungsten wire calorimeters [25] or one-dimensional carbon fiber-composite tile [26] placed at a short distance from the last grid to limit the beamlet overlap.

\section{Acknowledgements}

The authors very much appreciate the support of Dr. Antonio Pimazzoni for the initial studies on the IR technique here presented.

This work has been carried out within the framework of the EUROfusion Consortium and has received funding from the Euratom research and training programme 20142018 and 2019-2020 under grant agreement number 633053. The views and opinions expressed herein do not necessarily reflect those of the European Commission.

\section{List of the most relevant symbols}

\begin{tabular}{|c|c|}
\hline$\delta$ & Single beamlet width \\
\hline$a_{i}$ & $\begin{array}{l}\text { peak amplitude of the single Gaussian function } \\
\text { describing the beamlets }\end{array}$ \\
\hline$\vartheta$ & beamlet divergence \\
\hline$\sigma$ & Beamlet group width \\
\hline$y_{t o p} / y_{b o t}$ & Beam segment position vertical position \\
\hline$A_{i}$, with $i=1 \ldots 8$ & $\begin{array}{l}\text { Amplitude of each Gaussian function describing the } \\
\text { beamlet groups }\end{array}$ \\
\hline $\begin{array}{l}V o l_{t o p} / V o l_{b o t} \\
\varepsilon\end{array}$ & $\begin{array}{l}\text { Integral to infinity of the top/bottom beam segments } \\
\text { surface emissivity }\end{array}$ \\
\hline$\tau_{o p t}$ & ZnSe window transmissivity \\
\hline
\end{tabular}

\section{References}

[1] R S Hemsworth et al. "Overview of the design of the ITER heating neutral beam injectors". In: New Journal of Physics 19.2 (Feb. 2017), p. 025005.

[2] B. Schunke et al. "Overview of the negative ion based neutral beam injectors for ITER". In: Review of Scientific Instruments 87.2 (2016), p. 02C101.

[3] U. Fantz et al. "Towards 20 A negative hydrogen ion beams for up to 1 h: Achievements of the ELISE test facility (invited)". In: Review of Scientific Instruments 87.2 (2016), 02B307.

[4] R. Hemsworth et al. "Status of the ITER heating neutral beam system". In: Nuclear Fusion 49.4 (Mar. 2009), p. 045006.

[5] A. Masiello et al. EU development of the ITER Neutral beam injector and test facilities. 2012. 
[6] B. Heinemann et al. "Design of the half-size ITER neutral beam source for the test facility ELISE". In: Fusion Engineering and Design 84.2-6 (2009). Proceeding of the 25th Symposium on Fusion Technology(SOFT-25), pp. 915-922.

[7] P. Franzen et al. "Progress of the ELISE test facility: results of caesium operation with low RF power". In: Nuclear Fusion 55.5 (2015), p. 053005.

[8] P. Franzen and U. Fantz. "Beam Homogeneity Dependence on the Magnetic Filter Field at the IPP Test Facility MANITU". In: AIP Conference Proceedings 1390.1 (2011), pp. 310-321.

[9] R. S. Hemsworth et al. "Status of the Development of the SINGAP Accelerator for ITER". In: AIP Conference Proceedings 925.1 (2007), pp. 290-305.

[10] R. Gutser et al. "Simulations for the generation and extraction of negative hydrogen ions in RF-driven ion sources". In: AIP Conference Proceedings 1097.1 (2009), pp. 297-306.

[11] T. Kalvas et al. "IBSIMU: A three-dimensional simulation software for charged particle optics". In: Review of Scientific Instruments 81.2 (2010), 02B703.

[12] Niek Den Harder. Private communication. Aug. 2019.

[13] U. Fantz et al. "Achievement of the ITER NBI ion source parameters for hydrogen at the test facility ELISE and present Status for deuterium". In: Fusion Engineering and Design 156 (July 2020), p. 111609.

[14] R. Nocentini et al. "Advanced ion beam calorimetry for the test facility ELISE". In: AIP Conference Proceedings 1655.1 (2015), p. 060006.

[15] R. Nocentini et al. "Beam diagnostic tools for the negative hydrogen ion source test facility ELISE". In: Fusion Engineering and Design 88.6-8 (2013), pp. 913917.

[16] A. BenIsmail et al. "Space charge compensation studies of hydrogen ion beams in a drift section". In: Phys. Rev. ST Accel. Beams 10 (7 July 2007), p. 070101.

[17] A. Hurlbatt et al. "The particle tracking code BBCNI for negative ion beams and its application to BATMAN upgrade". In: AIP Conference Proceedings 2052.1 (2018), p. 040007.

[18] B. Heinemann et al. "Upgrade of the BATMAN test facility for H- source development". In: AIP Conference Proceedings 1655.1 (2015), p. 060003.

[19] U. Fantz et al. "Advanced NBI beam characterization capabilities at the recently improved test facility BATMAN Upgrade". In: Fusion Engineering and Design (Dec. 2018).

[20] Dirk Wünderlich. Private communication. May 2019.

[21] Marco Barbisan et al. "Modeling and design of a beam emission spectroscopy diagnostic for the negative ion source NIO1". In: The Review of scientific instruments 85 (Feb. 2014), 02A708. 
[22] B. Zaniol. "Error evaluation in the spectroscopic measurement of high power beam angular divergence". In: Journal of Quantitative Spectroscopy and Radiative Transfer 112.3 (Feb. 2011), pp. 513-518.

4 [23] A. Pimazzoni. "Investigation of ELISE beam properties by means of the diagnostic $5 \quad$ calorimeter." Master Thesis. Universitá degli Studi di Padova, 14th Sept. 2014.

6 [24] Roger Barlow. Statistics. Wiley John + Sons, 26th July 1989.

7 [25] R. Nocentini et al. "A new tungsten wire calorimeter for the negative ion source $8 \quad$ testbed BATMAN Upgrade". In: Fusion Engineering and Design (2019).

9 [26] A. Pimazzoni et al. "Thermal characterization of the SPIDER diagnostic calorimeter". In: AIP Conference Proceedings 2052.1 (2018), p. 040012. 